\title{
Teoria e Método para um Modelo de Avaliação dos Hospitais de Ensino no Brasil
}

\author{
Theory and Method of an Evaluation \\ Model for Brazilian Teaching Hospitals
}

PALAVRAS-CHAVE

- Hospitais de Ensino;

- Avaliação;

- Métodos;

- Educação Médica.

\section{KEYWORDS}

- Teaching Hospitals;

- Evaluation;

- Methods;

- Medical Education.

\begin{abstract}
RESUMO
Hospitais de Ensino servem de cenário de prática para atividades curriculares de cursos da área da saúde, podendo ser de propriedade de uma Instituição de Ensino Superior, pública ou privada, ou formalmente conveniados a esta, apresentando como eixos de reestruturação atenção à saúde, gestão hospitalar, ensino e pesquisa. Desta forma, este trabalho tem como objetivo descrever um modelo heurístico de avaliação de Hospitais de Ensino com base nos conceitos de paradigma de pesquisa e de modelo teórico, tomando como hipótese de trabalho que a avaliação é uma aplicação da metodologia das ciências sociais. Assim, com respeito ao marco metodológico, elegemos a Avaliação de Quarta Geração de Egon G. Guba e Yvona S. Lincoln como metodologia de apreensão dos significados, valores, questões e preocupações frente aos processos de gestão, ensino, pesquisa e assistência, por meio de seus stakeholders, implicados no processo.
\end{abstract}

\section{ABSTRACT}

Teaching hospitals provide a practical setting for curricular activities of health courses. These hospitals might be owned by a public or private higher education institution, or may have a formal relationship with such a school, based on the axes of restructuring health care, hospital management, education and research. This work aims to describe a heuristic evaluation model for teaching hospitals based on research paradigm concepts and a theoretical model, and working on a hypothesis that the evaluation is an application of social science methodology. Therefore, as regards the methodological reference, we elect Egon G. Guba and Yvona S. Lincoln's Fourth Generation Evaluation as the methodology for ascertaining the meanings, values, issues and concerns in view of the management, teaching, research and assistance processes, by means of the stakeholders involved. 


\section{INTRODUÇÃO}

No contexto brasileiro, os Hospitais de Ensino (HE) são equipamentos relevantes por terem como pressupostos a atenção à saúde de alta complexidade, a formação de profissionais de saúde para o Sistema Único de Saúde (SUS) e o desenvolvimento de pesquisa e tecnologias para o setor. A Portaria Interministerial MEC/MS nº 1.000/2004 os define como Hospitais Gerais e/ou Especializados que servem de cenário de prática para atividades curriculares de cursos da área da saúde, podendo ser de propriedade de uma Instituição de Ensino Superior (IES), pública ou privada, ou formalmente conveniados a esta $^{1}$. Na literatura internacional, a definição desses hospitais tem conotação semelhante, uma vez que, segundo Grosskopf et al., apud Lobo et al. ${ }^{2}$, trata-se de instituições que possuem residentes e/ou são afiliadas a conselhos ou associações governamentais de ensino médico.

Em 2003, visando à integração das atividades de assistência, ensino e pesquisa às políticas prioritárias do SUS, o Ministério da Saúde estabeleceu a Secretaria de Gestão do Trabalho e Educação em Saúde (SGTES), relacionada à ordenação de recursos humanos em saúde, e o Departamento de Ciência e Tecnologia, responsável pelo desenvolvimento de pesquisas em saúde. Todavia, uma integração mais orgânica entre essas áreas só se tornou possível após a implementação da Política de Reestruturação dos Hospitais de Ensino ${ }^{2}$. Este programa, instituído em 2004, objetiva aprimorar e inserir os HE no SUS por meio de um contrato de gestão entre os gestores hospitalar e municipal de saúde, tendo como base: (1) a redefinição do papel dessas unidades no sistema municipal de saúde ou de referência; (2) a qualificação da inserção desses hospitais na rede de saúde municipal/estadual; (3) as definições dos mecanismos de referência e contrarreferência; (4) a mudança de estratégias de atenção; (5) a humanização da atenção à saúde; (6) a melhoria da qualidade dos serviços prestados; (7) a qualificação da gestão hospitalar; (8) o desenvolvimento de ações de educação permanente e de pesquisa de interesse do sistema de saúde vigente ${ }^{3}$.

Assim, para serem certificados como HE, os hospitais devem se submeter a um processo de avaliação paritária pelos ministérios da Saúde e da Educação, pautado nas seguintes exigências estabelecidas pela Portaria Interministerial $n^{\circ}$ 2.400, de 2 de outubro de 2007: (1) estar inscritos no Cadastro Nacional de Estabelecimentos de Saúde (CNES); (2) servir de campo de prática para atividades curriculares na área da saúde; (3) abrigar alunos em atividades curriculares de ao menos um curso de Medicina e de dois outros cursos da saúde; (4) possuir programas de residência médica credenciados pela Comissão Nacional de Residência Médica (CNRM); (5) garantir acompanhamento diário por docente ou preceptor para os estudantes de graduação e residentes; (6) dispor de projeto institucional próprio ou vinculado a IES para o desenvolvimento de atividades regulares de pesquisa científica e avaliação de tecnologias, de mecanismos de gerenciamento das atividades de ensino e de pesquisa, de instalações adequadas de ensino e de acesso a biblioteca atualizada e especializada na área da saúde; (7) participar das políticas prioritárias do SUS; (8) colaborar ativamente na constituição de uma rede de cuidados progressivos à saúde; (9) dedicar um mínimo de $60 \%$ dos leitos ativos ao SUS 4 .

Uma vez certificados, esses hospitais pactuam metas quantitativas e qualitativas, com suas respectivas secretarias de saúde, referentes a gestão, atenção à saúde, políticas prioritárias do SUS e ensino e pesquisa, por meio de um convênio. Essas metas são acompanhadas e avaliadas por uma Comissão Permanente de Acompanhamento de Contratos, composta por representantes da secretaria contratante, do hospital, dos docentes e discentes e do conselho local de saúde.

Entretanto, embora a contratualização permita o monitoramento e avaliação desses hospitais, por meio das comissões de acompanhamento do contrato, ainda se discutem os instrumentos para avaliar o impacto dessa política nessas unidades hospitalares e, de modo mais amplo, no sistema de saúde ${ }^{5}$. Ao realizarem uma revisão sistemática sobre avaliação de Hospitais de Ensino no Brasil, Nogueira et al. ${ }^{6}$ concluíram que a produção sobre essa temática corresponde a $8,6 \%$ das produções sobre HE e que, nos estudos, predominou o uso de métodos quantitativos, com recurso às ferramentas Análise de Dados Envoltória (DEA), Índice de Malmquist e Metodologia Multicritério de Apoio à Decisão (MCDA), próprios das abordagens positivistas que Guba e Lincoln denominaram Avaliação de Primeira Geração ${ }^{7}$.

Reconhecendo a evolução do campo da Avaliação, outras abordagens avaliativas podem ser úteis à tarefa de avaliar os HE. Nesse sentido, este estudo visa descrever a aplicação teórico-metodológica do Modelo Responsivo e Construtivista proposto por Guba e Lincoln ${ }^{7}$ à avaliação dos HE no Brasil.

\section{ESQUEMA CONCEITUAL}

Avaliar é, certamente, um ato próprio do processo de cognição do ser humano. Representa uma tomada de posição frente ao vivido, um julgamento subjetivo acerca da realidade, o engendramento de um novo devir para a nossa existência. No entanto, se deslocarmos essa atividade humana própria do senso comum para o âmbito da cognição cientificamente estrutura$\mathrm{da}$, teremos de buscar nos fundamentos da racionalidade científica a coerência lógico-semântica que determina a tomada de posição frente ao vivido, o julgamento e o engendramento do devir como cientificamente válidos, ou seja, como dotados de convicção de validade universalmente reconhecida. Nesse 
sentido, a realidade sob avaliação visada pela ciência, traduzida pelos objetos da pesquisa avaliativa, deve abarcar tanto a descoberta quanto a prova reflexiva.

Neste trabalho, faremos a descrição de um modelo heurístico de avaliação de $\mathrm{HE}$ com base nos conceitos de paradigma e de modelo, indo buscar na reflexão de Domingues ${ }^{8}$ sobre a epistemologia das ciências humanas a delimitação de ambos os conceitos que empregaremos neste estudo, constituindo-se em seu esquema conceitual. $\mathrm{O}$ autor propôs restringir o termo paradigma a seu uso intelecto-científico, distinguindo-o da palavra modelo. Ele coloca os termos paradigma e teoria como aproximados e integra o termo modelo à operacionalização do método. Tal abordagem, na ótica do autor, é necessária para atender à delimitação do fazer científico, que comportaria duas dimensões: (1) uma dimensão teórica, em cujo interior se formula o problema a ser investigado, se postula algo a respeito da realidade, se elege uma parte ou segmento do real como elemento ou princípio explicativo; e (2) uma dimensão metódica, instalada pela teoria e guiada por ela, a qual se encarregará, entre outras coisas, de contrastar a teoria em relação à realidade, servindo-se de técnicas e procedimentos apropriados.

Contudo, paradigma e teoria são conceitos distintos. Para De Bruyne et al. ${ }^{9}$, o paradigma exerce uma função de vigilância crítica, garantindo a produção do objeto científico e da explicitação das problemáticas da pesquisa. Encarrega-se de renovar continuamente a ruptura dos objetos científicos com os do senso comum e decide, em última instância, sobre as regras de produção e de explicação dos fatos, e sobre a compreensão e a validade das teorias. A teoria, por seu turno, é o lugar da formulação sistemática dos objetos científicos, visando à sua inteligibilidade.

Já o termo modelo, para Domingues ${ }^{8}$, designa, numa acepção tecnológico-científica, uma ferramenta teórica ou um instrumento analítico de que o cientista se serve para orientar suas pesquisas, assim como para contrastar o real empírico e o que se pensa sobre ele. Com efeito, são modelos científicos, em sua qualidade de ferramentas de conhecimento, todo um conjunto de esquemas, de diagramas, de tipologias e de outros tantos artifícios por meio dos quais os cientistas simulam o real, permitindo controlar a teoria, verificar suas hipóteses e submeter suas predições a testes precisos.

Para atingirmos o objetivo deste estudo, seguindo Aguilar e Ander-Egg ${ }^{10}$, tomaremos como hipótese de trabalho que a avaliação é uma aplicação da metodologia das ciências sociais, que Scriven ${ }^{11}$ denomina Modelo das Ciências Sociais de Avaliação. Nesse sentido, tomaremos o Construtivismo como paradigma de avaliação - caracterizado por uma ontologia relativista, por uma gnosiologia subjetivista e por uma metodologia hermenêutica e dialética ${ }^{12}-$, a partir do qual se constituiu a Avaliação de Quarta Geração ${ }^{7}$, que, incorporando a delimitação normativa brasileira dos HE, será tomada como modelo de avaliação destes equipamentos de assistência, ensino e pesquisa.

\section{O Hospital de Ensino no Brasil: delimitação normativa}

A Constituição Federal de 1988, ao criar o SUS, estatui em seu Artigo 200 que, além de outras atribuições, compete a ele a ordenação da formação de recursos humanos na área da saúde, regulada ainda pela Lei Orgânica de Saúde 8.080/90, em seu Artigo 6º.

A partir desse arcabouço jurídico, foi necessário implementar uma política que fomentasse uma reestruturação das instituições hospitalares, com enfoque nos hospitais universitários e de ensino (HUE), a fim de responder às necessidades de saúde da população de forma integrada às redes de serviços de saúde local e regional. No período em que se delineava a reformulação da saúde brasileira, aos HUE era atribuído um Índice de Valorização de Desempenho (IVD), por meio da Portaria Interministerial MEC/MPAS no 15 de 1987, no âmbito do Convênio MEC/MPAS vigente ${ }^{3}$.

Esse IVD propunha gerar recursos para a expansão e aperfeiçoamento das próprias atividades hospitalares a partir do Índice de Valorização Hospitalar (IVH), definido pelos critérios de complexidade, resolutividade, integração à rede de serviços e perfil dos recursos humanos, e pelo Índice Adicional de Valorização (IAV), de acordo com as atividades desenvolvidas pelo hospital.

Contudo, esta política falhou em dois aspectos: não houve detalhamento qualitativo e quantitativo dos requisitos para os hospitais receberem os respectivos índices de desempenho, e não houve acompanhamento e avaliação adequados ${ }^{3}$. Isto levou à extinção desses índices, por meio da Portaria MS/ SNAS no 15, de janeiro de 1991, bem como à criação do Fator de Incentivo ao Desenvolvimento do Ensino e Pesquisa Universitária em Saúde (Fideps). De acordo com essa portaria, são Hospitais Universitários aqueles reconhecidos pelo Ministério da Educação (MEC), funcionando há mais de cinco anos e participantes de algum Sistema Integrado de Procedimentos de Alta Complexidade (Sipac) do Ministério da Saúde (MS).

Em 1994, são estabelecidos novos critérios para a concessão do Fideps, mediante a Portaria MS/MEC nº 1, de 16 de agosto, sendo acrescidos percentuais de $25 \%, 50 \%$ ou $75 \%$ sobre a receita das internações. Por conseguinte, após cinco anos dessa portaria, o MS estabelece, por meio da Portaria MS no 1.127, de agosto de 1999, que as secretarias de saúde que tenham sob sua gestão hospitais habilitados ao recebimento do Fideps procedam à verificação in loco das condições de funcionamento dos mesmos. Ademais, conforme a Portaria MS n ${ }^{-}$ 1.480 , de dezembro de 1999, o montante de recursos repassa- 
dos mensalmente estaria determinado no Contrato de Metas. Entretanto, esses recursos não serviram para estimular ou fortalecer as atividades de ensino e pesquisa nesses hospitais, já que eram incorporados ao custeio dessas instituições a fim de contornar problemas de financiamento de natureza variada ${ }^{3}$.

Neste aspecto, em 2003, foi instituída uma Comissão Interinstitucional, por meio da Portaria MS/MEC/MCT/MPOG nº 562, que tinha por objetivo avaliar e diagnosticar a situação dos HUE no Brasil, para reorientar e/ou reformular a política para o setor, tendo como resultado a formulação de proposta de reestruturação dos HE por meio da certificação e da contratualização.

Desta forma, com vistas à identificação dessas unidades hospitalares, as Portarias Interministeriais MEC/MS nº 1.000 e no 1.005 , de 2004, apresentam definições e critérios para certificação dos HE, enfatizando que o processo de certificação é de responsabilidade conjunta dos ministérios da Saúde e da Educação. Assim, é implantado o Programa de Reestruturação dos Hospitais de Ensino do Ministério da Educação no SUS, por meio da Portaria Interministerial MS/MEC n⿳0 1.006, de 2004, bem como o Programa de Reestruturação dos Hospitais de Ensino no âmbito do SUS, por meio da Portaria Interministerial MS/MEC no 1.702 , de 2004, sendo o repasse de recursos financeiros a essas unidades hospitalares definido pelas Portarias MS/GM no 1.107, 1.703 e 2.352. de 2004.

No ano de 2007, a Portaria Interministerial MS/MEC no 2.400 estabelece novos requisitos para a certificação de unidades hospitalares como Hospital de Ensino, revogando as Portarias Interministeriais $n^{\circ} 1.000$ e 1.005, de 2004. Entre as principais mudanças, consta a exigência de que a unidade hospitalar seja de propriedade de Instituição de Ensino Superior (IES), pública ou privada, ou que esteja formalmente conveniada a uma IES, além de se destacar que deve ser campo de prática de dois cursos da área da saúde, além da Medicina.

Esse arcabouço jurídico-normativo propõe a reestruturação desses hospitais com base em três grandes eixos: (1) ações estratégicas na área da assistência; (2) gestão hospitalar, ensino e pesquisa; (3) mudança do modelo de alocação de recursos financeiros, por meio do processo de contratualização. Esta seria o meio pelo qual o representante legal do hospital de ensino e o gestor local do SUS estabelecem metas quantitativas e qualitativas relacionadas a atenção à saúde, ensino e pesquisa, e gestão hospitalar. Tais metas devem ser acompanhadas e atestadas pelo Conselho Gestor da Instituição ou pela Comissão Permanente de Acompanhamento de Contratos ${ }^{3}$.

Tendo em vista essa perspectiva normativa, é necessário estabelecer propostas metodológicas de avaliação dos HE como estratégia de ordenamento da formação de recursos humanos em saúde no âmbito do SUS. Propomos, como resposta, a metodologia da Avaliação de Quarta Geração de Guba e Lincoln?
A abordagem metodológica responsiva e construtivista da Avaliação de Quarta Geração

Considerando que o modelo é a instância que enuncia as regras de estruturação, de formação do objeto científico, impondo-lhe certa figura, certa ordem entre seus elementos, colhemos do campo da avaliação de programas um modelo avaliativo capaz de operacionalizar a apreensão do desempenho dos HE.

Guba e Lincoln? ${ }^{7}$, seguindo a abordagem avaliativa responsiva de $S_{\text {take }}{ }^{13}$ e a abordagem naturalista de investigação que eles mesmos propuseram ${ }^{14}$, elaboraram um modelo que denominaram Avaliação de Quarta Geração, que se insere numa tipologia geracional de abordagens metodológicas em avaliação, criada por eles. Tal modelo se caracteriza por uma avaliação inclusiva e participativa, que é feita como um processo de negociação entre os atores implicados na intervenção a ser avaliada (stakeholders). Em outras palavras, é um processo interativo negociado, que se fundamenta num paradigma construtivista, cuja tarefa é reorientar o paradigma positivista, hegemônico nas ciências sociais e no campo da Avaliação.

A Avaliação de Quarta Geração é uma alternativa aos referenciais anteriores: (1) a Avaliação de Primeira Geração, que se caracteriza pela mensuração; (2) a Avaliação de Segunda Geração, que se caracteriza pela descrição; (3) a Avaliação de Terceira Geração, que se caracteriza pelo julgamento de mérito e valor. Estas gerações apresentam graves problemas e limitações: (1) tendência à supremacia do ponto de vista gerencial nos processos avaliativos; (2) incapacidade em acomodar o pluralismo de atores envolvidos em torno de qualquer programa; (3) hegemonia do paradigma positivista, cujo questionamento aponta a discussão sobre os enfoques antropológicos e socioeconômicos na análise crítica de programas ou serviços, que representam desafios metodológicos para o campo da avaliação.

O núcleo operacional desse modelo de avaliação é o círculo hermenêutico dialético (Figura 1), que é integrado por respondentes (os implicados no programa ou stakeholders), codificados com a letra " $\mathrm{R}$ ", a partir dos quais são obtidas construções, codificadas com a letra " $\mathrm{C}$ ". Ele se inicia com o respondente 1 (R1), selecionado pelo investigador a partir de indicação por um informante-chave ou por sua privilegiada posição no contexto de investigação. Este respondente é submetido a uma entrevista para determinar uma construção êmica inicial do que está sendo investigado ou avaliado (o enfoque da investigação). O respondente é solicitado a descrever e comentar o enfoque tal como o constrói, em seus próprios termos. Essa descrição e comentários podem incluir observações sobre reivindicações, preocupações e questões sobre o objeto avaliado, bem como sobre o que é ou não pertinente em relação ao objeto avaliado. 


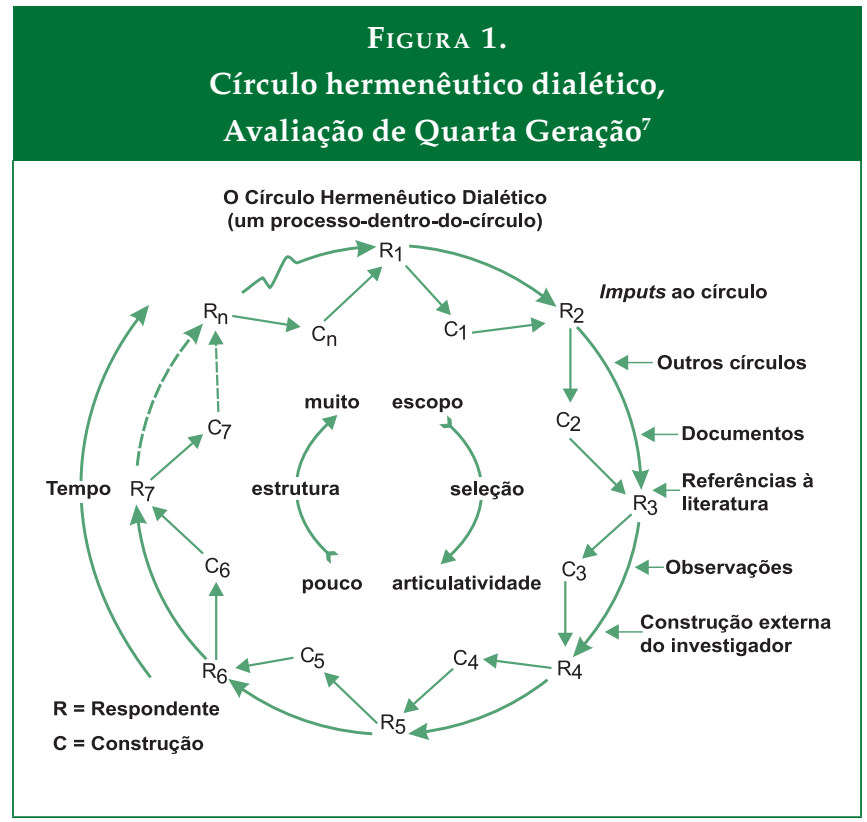

Em seguida, o respondente R1 é solicitado a nominar outro respondente, $\mathrm{R} 2$, que tem uma construção diferente da sua, tanto quanto the é possível identificar. Os temas, conceitos, ideias, valores, preocupações e questões centrais propostos pelo respondente R1 são analisados pelo investigador como uma formulação inicial da construção de R1, designada como C1. O processo de análise de dados, no círculo hermenêutico dialético, é o método da comparação constante. Ele segue de perto o momento da coleta de dados (a abordagem dos respondentes) e é completado para R1 antes da abordagem a R2. Se os temas à mão na abordagem a R1 são de grande importância, pode ser útil checar a análise completada com R1, para assegurar-se de que o investigador interpretou corretamente a construção de R1.

Depois, R2 é entrevistado, sendo-lhe permitida a mesma liberdade de expressão dada a R1. Quando, porém, R2 tiver livremente colocado a sua construção, os temas da análise de R1 são introduzidos, e R2 é convidado a comentá-los. Como resultado da entrevista com R1, é produzida informação não

\section{QuADro 1.
PASSO
Etapas da Metodologia da Avaliação de Quarta Geração}

1. Contratualização

2. Organização

3. Identificação dos implicados no programa avaliado (stakeholders)

4. Desenvolvimento de construções conjuntas dentro de grupos

5. Alargamento das construções conjuntas dos implicados no programa (stakeholders) por meio de novas informações e do aumento da sofisticação

6. Classificação das reivindicações, preocupações e questões solucionadas por consenso

7. Priorização dos itens não solucionados por consenso

8. Coleta e sofisticação da informação

9. Preparação da agenda para a negociação

10. Condução da negociação

11. Compilação do relatório

12. Retomada
Iniciar contrato com cliente

Selecionar e treinar a equipe de avaliação; fazer arranjos para a entrada em campo; fazer arranjos logísticos e acessar fatores políticos locais

Identificar os implicados no programa (stakeholders); montar estratégias de pesquisa contínuas; obter e formalizar condições de concordância em participar da pesquisa (submetendo o protocolo a um comitê de ética em pesquisa)

"Rodar" os círculos hermenêuticos dialéticos para fazer emergir as construções conjuntas; checar credibilidade

Refazer os círculos hermenêuticos dialéticos, utilizando informação documental, interpolação de entrevistas de esclarecimento e de observações, referências bibliográficas e a construção externa (ética) dos investigadores

Identificar as reivindicações, preocupações e questões solucionadas por consenso; deixar de lado como componentes de relatórios de casos

Determinar processo de priorização participativo; submeter itens para priorização; checar credibilidade

Coletar informação e treinar negociadores no seu uso por meio de: utilização de novos círculos hermenêuticos dialéticos, obtenção de informação existente, utilização de novos instrumentos ou de instrumentos existentes e realização de novos estudos

Definir e elucidar itens não solucionados; elucidar construções que competem entre si; iluminar, apoiar e refutar itens; prover sofisticação no treinamento; testar agenda

Selecionar círculo hermenêutico dialético representativo; "rodar" o círculo; moldar a construção conjunta; checar credibilidade; determinar a ação

Compilar relatórios dos grupos de envolvidos no programa (stakeholders); compilar o relatório do caso

Retomar todo o processo 
somente sobre R2, mas também uma crítica da construção a partir de R1.

$\mathrm{O}$ investigador solicita a $\mathrm{R} 2$ a nominação de um respondente R3 e completa a segunda análise, resultando na formulação de C2, uma nova, mais informada e sofisticada construção baseada em duas fontes, R1 e R2. Este é o início da construção conjunta dentro de um grupo.

O processo é repetido com novos respondentes sendo integrados ao círculo até que a informação coletada se torne redundante ou caia em duas ou mais construções que permanecem em conflito.

Tal como pode ser observado na Figura 1, a obtenção de informações relevantes para a avaliação é obtida de um processo interativo, podendo-se adicionar às informações emergentes da "rodagem" do círculo informações obtidas externamente a ele, como construções de círculos hermenêuticos dialéticos com outros implicados no programa (stakeholders), análise de documentos, referências à literatura especializada, observações e a construção externa (ética) do investigador.

Os círculos hermenêuticos dialéticos integram o fluxo de 12 etapas da metodologia da Avaliação de Quarta Geração, tal como determinadas por Guba e Lincoln ${ }^{7}$ e listadas no Quadro 1.

O modelo de avaliação aqui proposto combina conscientemente as perspectivas da normatização governamental brasileira concernente aos HE e da abordagem responsiva e construtivista de avaliação. E essa combinação se dá no conceito de stakeholder, que é aquele que tem investimento pessoal, credibilidade, poder, futuro ou outro capital investido no programa e que, então, pode estar sob algum grau de risco com ele ${ }^{10}$.

Guba e Lincoln ${ }^{7}$ identificam, no âmbito da Avaliação de Quarta Geração, três grandes classes de atores implicados na intervenção sob avaliação: (1) os agentes, aquelas pessoas envolvidas em produzir, usar e implementar a intervenção avaliada; (2) os beneficiários, aqueles que tiram proveito de alguma forma do uso da intervenção avaliada; (3) as vítimas, pessoas negativamente afetadas pelo uso da intervenção avaliada. Entendemos que a abordagem responsiva e construtivista de Guba e Lincoln ${ }^{7}$ é aplicável à captação dos sentidos sobre a realidade, produzidos pelos stakeholders dos HE. Os círculos representativos desses stakeholders seriam: (1) da gestão do SUS, do hospital e das escolas médicas; (2) das comissões de avaliação dos Hospitais de Ensino; (3) dos docentes e preceptores; (4) dos discentes (graduação e residência); (5) dos profissionais de saúde. Eles podem ser organizados e "rodados", e as construções de ambos os círculos serviriam como input de uns para os outros como forma de solucionar por consenso as reivindicações, as preocupações e as questões emergentes. No processo de "rodagem" dos círculos, outros inputs podem ser utilizados, a depender das construções obtidas e dos stakeholders identificados.

Esse modelo de avaliação está inserido no cumprimento de todas as etapas preconizadas no Quadro 1. O processo avaliativo conclui-se na décima primeira etapa, com a compilação do relato de caso, embora a etapa 12 possa ser continuada, incluindo-se novos círculos hermenêuticos e dialéticos, a partir da negociação feita na etapa 10 do processo avaliativo empreendido.

\section{CONCLUSÃO}

Existem aspectos epistemológicos essenciais a serem abordados quanto ao modo de se obter conhecimento válido sobre o desempenho dos HE numa pesquisa avaliativa.

Com relação ao modo de se obter conhecimento válido sobre o objeto avaliado, sugerimos a necessidade de partir de um novo marco epistemológico, capaz de dar conta da análise dos aspectos microssociais e macrossociais inerentes aos processos de gestão, pesquisa e extensão próprios de um HE, assinalando a insuficiência do paradigma hegemônico de investigação em avaliação desses equipamentos, presente na literatura, e a pertinência de um pensar complexo sobre o seu desempenho.

Quanto ao modelo teórico que define os HE como objeto avaliado, elegemos, a partir da sua base normativa, os eixos: gestão hospitalar, políticas prioritárias, ensino e pesquisa, e atenção à saúde. Com respeito ao modelo teórico sobre como avaliar os HEs, elegemos a Avaliação de Quarta Geração como metodologia de apreensão de significados, valores, questões e preocupações frente aos processos de gestão, ensino, pesquisa e assistência, por intermédio de seus stakeholders, implicados no processo.

\section{REFERÊNCIAS}

1. Brasil. Portaria Interministerial 1.000 de 15 de abril de 2004 [capturado 10 out. 2011]. Disponível em: http:// www.femerj.org.br/Boletim/Federal/Ministerio\%20Saude/GM/2004/Junho/PORTARIA\%20INTERMINISTERIAL\%20N\%BA\%201000.pdf.

2. Lobo MSC, Lins MPE, Silva ACM, Fizman R. Avaliação de desempenho e integração docente-assistencial nos hospitais universitários. Rev. Saúde Pública [online]. 2010. 44 (4) [capturado 30 mai. 2013]; 581-590. Disponível em: http: / / www.scielo.br/scielo.php?script=sci_arttext\&pid =S0034-89102010000400001.

3. Brasil. Ministério da Saúde. Secretaria de Atenção à Saúde. Departamento de Atenção Especializada. Sociedade Hospital Samaritano. Política de Reestruturação dos Hospitais 
de Ensino e Filantrópicos no Brasil no período de 20032010: uma análise do processo de implantação da contratualização. Brasília (DF); 2012.

4. Brasil. Portaria Interministerial no 2.400 de 02 de outubro de 2007. Disponível em: http://www.saude.mt.gov.br/ upload/legislacao/2400-\%5B2830-120110-SES-MT\%5D. pdf Acessado em: 2011 out 10.

5. Lins ME, Lobo MSC, Silva ACM, Fiszman R, Ribeiro VJP. O uso da análise envoltória dos dados (DEA) para avaliação de hospitais universitários brasileiros. Rev. Ciência \& Saúde Coletiva [online]. 2007. 12(4) [capturado 29 mai. 2013]; 995-998. Disponível em: http:/ / www.scielo.br/scielo.php?pid=S1413-81232007000400020\&script=sci_arttext

6. Nogueira DL, Lira GV, Albuquerque IMN, Linhares MSC. Avaliação dos Hospitais de Ensino no Brasil: uma revisão sistemática. Rev. Bras. Educ. Méd. 2015 [no prelo].

7. Guba EG, Lincoln YS. Fourth Generation Evaluation. Newbury Park: SAGE Publications; 1989. 294 p.

8. Domingues I. Epistemologia das ciências humanas (tomo 1): positivismo e hermenêutica. São Paulo: Edições Loyola; 2004. $671 \mathrm{p}$.

9. De Bruyne P, Herman J, De Schoutheete M. Dinâmica da pesquisa em ciências sociais. Rio de Janeiro: Francisco Alves; 1977.

10. Aguilar MJ, Ander-EGG E. Avaliação de serviços e programas sociais. Petrópolis: Vozes; 1994. 199 p.

11. Scriven M. Evaluation thesaurus. $4^{\text {th }}$ ed. Newbury Park: SAGE Publications; 1991.391 p.
12. Guba E. The alternative paradigm dialog. In: Guba E (Ed.). The paradigm dialog. Newbury Park: SAGE Publications; 1990. p. 17-30.

13. Stake R. Program evaluation, particularly responsive evaluation. In: Stufflebeam DL, Madaus GF, Kellaghan, T. (Eds). Evaluation models: viewpoints on educational and human services evaluation. $2^{\text {nd }}$ ed. Norwell: Kluwer Academic Publishers, 2000, p. 343-362.

14. Lincoln YS, Guba E. Naturalistic inquiry. Newbury Park: Sage Publications, 1985, 416 p.

\section{CONTRIBUIÇÃO DOS AUTORES}

Geison Vasconcelos Lira contribuiu com o desenho do estudo, elaboração da versão final e revisão crítica para a publicação. Denise Lima Nogueira contribuiu substancialmente para o desenho do estudo e elaboração da versão final para a publicação.

\section{CONFLITO DE INTERESSES}

Declarou não haver.

\section{ENDEREÇO PARA CORRESPONDÊNCIA}

Geison Vasconcelos Lira

Rua Geraldo Rangel, 186

Derby - Sobral

CEP 62041-040 - CE

E-mail: vasconlira@gmail.com 